\title{
Impacted third molar transplantation on the malpracticed extraction socket
}

\author{
Soung Min Kim ${ }^{1,2}$ and Emmanuel K. Amponsah ${ }^{1}$ \\ Ghana Med J 2017; 51(4): 200-203 DOI: http://dx.doi.org/10.4314/gmj.v51i4.10
}

${ }^{1}$ Oral and Maxillofacial Microvascular Reconstruction LAB, Brong Ahafo Regional Hospital, Sunyani, Ghana, ${ }^{2}$ Department of Oral and Maxillofacial Surgery, Dental Research Institute, School of Dentistry, Seoul National University, Seoul, Korea

Corresponding author: Prof. Soung Min Kim

E-mail: smin5@snu.ac.kr

Conflict of interest: None declared

\section{SUMMARY}

Autotransplantation with or without endodontic therapy is regarded as an alternative treatment option for the replacement of missing teeth. A primary responsibility of a maxillofacial surgeon is to reverse any malpractice to promote successful outcomes and improve the patient's quality of life. This paper presents a malpractice case of incorrect extraction of the lower second molar instead of the impacted third molar. A simple technique of transplanting the impacted third molar to the site of the extracted second molar is introduced by a maxillofacial specialist in Ghana. By making an intentional root socket and fixation without using additional appliances, a novel second molar was achieved with complete recovery. This patient was followed after transplant for a four-year period with the best satisfaction.

Funding: A grant of the Korean Health Technology R\&D Project, Ministry of Health \& Welfare, Republic of Korea. (HI15C0689)

Keywords: Autotransplantation, Endodontic treatment, Impacted third molar, Malpracticed extraction, Maxillofacial specialty

\section{INTRODUCTION}

Tooth autotransplantation is the transplantation of one's own tooth and adjacent tissues from one site of the mouth to another edentulous site. Replantation of a tooth has also been defined as the surgical reattachment of a tooth that has been completely removed from a person's alveolar bone. Although tooth autotransplantation and replantation is considered a conventional treatment for tooth replacement, most dentists have emphasized the use of dental implants for missing tooth replacement. ${ }^{1}$

This clinical case report presents an autotransplantation in a 26-year-old patient who suffered from incorrect extraction of an intact lower second molar instead of the impacted third molar. This paper also reviews the basic techniques of creation of an intentional root socket and fixation without using additional appliances. Complete recovery from this improper extraction was achieved using this method.

\section{CASE REPORT}

A 26-year-old Korean female was referred to the Oral and Maxillofacial Department of Seoul National University Dental Hospital for the implant replacement of her missing right lower second molar. The right lower second molar has been erroneously extracted instead of her impacted third molar in a private dental clinic approximately six months prior.

She had non-pathologic gingivae surrounding the right lower first molar, and a small operculum was found at the site of the impacted third molar. She had no pain but slight discomfort in the area of the missing tooth. The patient was generally healthy without pregnancy or osteoporosis, but she looked very nervous and was disappointed with her local dentist for this malpractice. She had already visited several clinics that recommended implant therapy, and she finally visited our hospital for surgical extraction of the impacted third molar with combined implantation. 
Panoramic view revealed that impacted right lower third molar erupted horizontally (Figure 1B) compared with previous preextraction state of lower second molar in patient's bringing radiogram (Figure 1A).

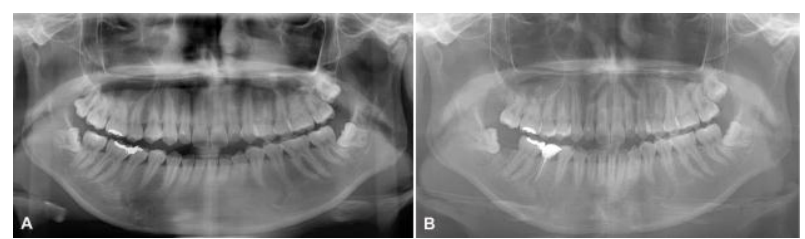

Figure 1 Preoperative panoramic views. The first was taken in a private clinic (A), and the second was taken in our hospital six months after extraction of the right lower second molar (B).

There were no other remarkable pathologic findings near the extraction socket, so we recommended an autotransplantation and implant fixture installation if autotransplantation failed. After initial consultation regarding autotransplantation of the impacted third molar to the missing second molar site, the patient decided to undergo the proposed treatment. Instead of implant installation combined with surgical extraction of the impacted third molar, the autotransplantation technique can be performed to improve the patient's quality of life. Our surgical plan consisted of the following: 1) intact extraction of the impacted third molar, 2) minimal retention of the mesial root in the extraction socket by drilling, 3) healing procedure in the extraction socket of the impacted third molar, and 4) direct endodontic therapy before autotransplantation. The patient agreed to our suggestions and understood the expected results.

Under local anaesthesia, a horizontal incision continuous with a circular incision around the first molar was created (Figure 2A). The impacted third molar was extracted without any damage to the tooth (Figure 2B), and it was inserted into the second molar site after making a small hole in the mesial root cavity of the extraction socket (Figures 2C, 2D). We decided not to perform a root canal of the donor tooth due to the patient's age and because the three current roots were all severely curved. We acquired rigid initial retention between the donor tooth and adjacent teeth without any splinting. An intentional resin splint was applied to the upper molars to prevent unnecessary extrusions before full recovery of the transplanted tooth (Figure 2D). After checking for occlusions (Figure 2D), figure eight sutures were tied around the donor tooth (Figure 2E). On postoperative radiograph, an acceptable seating position was confirmed (Figure 3A). After postoperative consultation, the patient maintained a soft and semi fluid diet with frequent hygienic care. After one week, the tooth remained stable in place with no signs of infection (Figures $2 \mathrm{~F}$ and $3 \mathrm{~B}$ ).

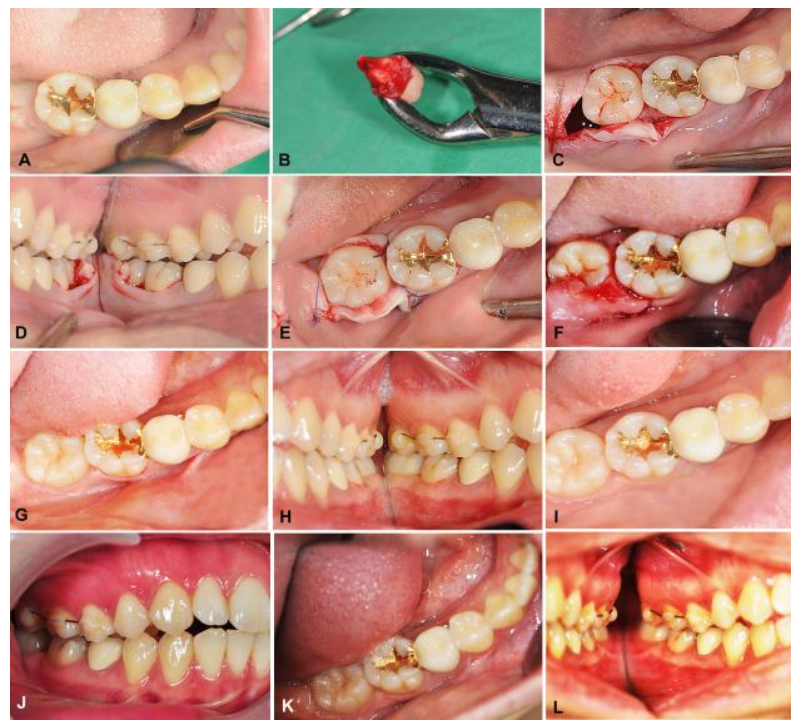

Figure 2 Impacted third molar transplantation procedures. Preoperative occlusal appearance (A), appearance of the extracted, impacted third molar with forceps (B), occlusal (C) and buccal biting (D) views of transposition to the lower second molar site after making a mesial root cavity, figure 8 suturing of the surrounding mucosa $(\mathrm{E})$, stitch out appearance after one week $(\mathrm{F})$, occlusal $(\mathrm{G})$ and buccal biting $(\mathrm{H})$ appearance after five months, occlusal (I) and buccal biting (J) appearance after 13 months, and occlusal (K) and buccal biting (L) appearance after three years.
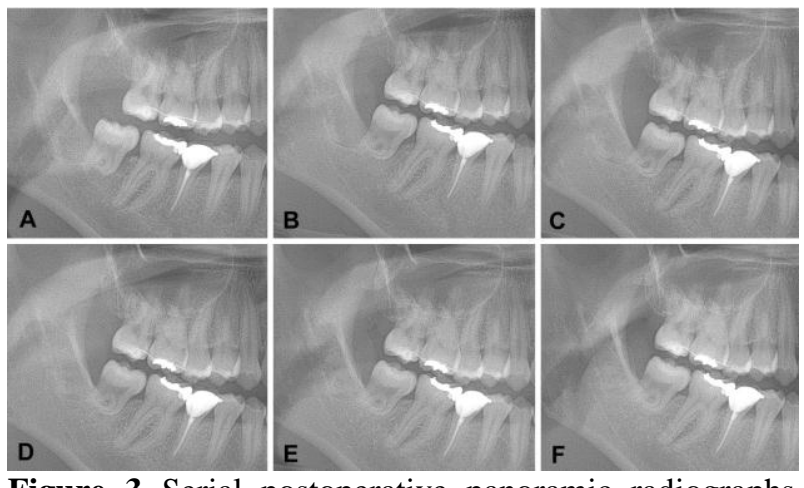

Figure 3 Serial postoperative panoramic radiographs from operation day (A), one week later (B), five months later (C), 13 months later (D), two years later (E), and three years later $(\mathrm{F})$. 
During follow-up visits at one month, five months (Figures 2G and 2H, Figure 3C), 13 months (Figures 2I, 2J, 3D), two years (Figure 3E), and three years (Figures $2 \mathrm{~K}, 2 \mathrm{~L}, 3 \mathrm{~F})$, the transplanted tooth naturally incorporated with the rest of her teeth.

\section{DISCUSSION}

Autotransplantation is an old treatment option for replacing missing teeth when only a donor tooth is available. More recently, instead of popular implant therapy, autotransplantation has been considered an ideal treatment, especially for a growing patient. ${ }^{1,2}$ Autotransplantation of a third molar to another molar site was also considered due to the benefits for the patient, and this technique can be highly successful if the appropriate patients are selected and if the basic principles of transplantation are followed. A case of intraalveolar transplantation using a simple extraction and repositioning with extrusion and rotation of the root to allow sufficient crown preparation in the case of crown-root fracture was previously reported. ${ }^{1,2}$ The success rate of autotransplantation was $60 \%$ in 10 cases of dentigerous cysts affecting permanent teeth. ${ }^{4}$ The predictable success and survival rates of autotransplanted premolars and molars compared to dental implants were greater than $90 \%$. A retrospective study of 97 consecutive young patients showed an $82 \%$ success rate and $98.2 \%$ presence during a 13.4-month median follow-up period. ${ }^{2}$

Usually, the success criteria of autotransplantation include clinical and radiographical findings that are assessed by univariate or multivariate analysis. A marginal gingival status and periapical status with adjacent alveolar bone could be considered as addition success criteria. In this case, we found a healthy gingiva and a well-placed tooth near the first molar (Figures $2 \mathrm{~K}$, $2 \mathrm{~L}$ ). Some portions of the periodontal ligament space were connected to the roots of the implanted tooth, and sufficient bony deposition in the bifurcation and surrounding area was observed on the three-year followup radiograph (Figure $3 \mathrm{~F}$ ).

A study ${ }^{3}$ showed that, if suitable donor teeth are available, autotransplantation is a viable treatment for missing teeth regardless of the age of the patient. Autotransplantation with complete root formation had an $84 \%$ success rate regardless of whether endodontic therapy was received before surgery or after transplantation. ${ }^{4}$ Pulp revascularization is a hallmark of successful case, while root resorption is considered a failed case. ${ }^{5}$ In this case, we did not perform a root canal on the donor tooth during transplantation due to the patient's age and because three roots were severely curved. To avoid time delay, root canal enlargement, and filling procedures, we inserted the donor tooth into the constructed socket while preserving the periodontal ligaments and bloody contents. The patient had also been considered for root canal therapy during her follow-up period, but she declined this procedure.

In this case, the recipient extraction socket had already healed during the six months following the erroneous extraction. As such, we created a root socket for the donor tooth. To create this root space, we used a round bur, 2.0- to 3.5-mm-diameter drills, and different shapes of countersinking drills to produce the tapered shape of the root cavity. Creating a root space for the distal root cavity was not necessary in this case due to the crown space of the impacted third molar. For more exact shape prediction, a rapid prototype model using threedimensional printing technology could also be considered, but we did not utilize this due to its high cost. For fixation of the donor tooth in the recipient site, several methods including resin wire splint, arch bar adaptation, thick nylon suture, and orthodontic bracket bonding were considered. Due to the tooth's good fit within the extraction socket, only firm pressure was needed for full fixation. (Figures 2A-D)

The results of one recent study showed that incorrect positioning with the absence of occlusal and interproximal contacts in the donor tooth led to the need for additional orthodontic treatment. The presence of occlusal contacts, temporary splint fixation of the upper teeth, and creating a mesial root cavity space for firm seating and tight interproximal contact to the distal surface of lower first molar likely contributed to the good outcome of this case. Without any splinting between the donor and adjacent teeth, we acquired rigid initial retention. An intentional resin splint was applied to the upper molars to prevent both unnecessary extrusions and severe occlusions before full recovery of the transplanted tooth (Figure 2D).

\section{ACKNOWLEDGEMENT}

This study was supported by a grant of the Korean Health Technology R\&D Project, Ministry of Health \& Welfare, Republic of Korea (HI15C0689) and by Basic Science Research Program through NRF of Korea(No. 2017R1D1A1B04029339).

\section{REFERENCES}

1. Mendes RA, Rocha G. Mandibular third molar autotransplantation--literature review with clinical cases. J Can Dent Assoc 2004;70:761-766. 
2. Verweij JP, Toxopeus EE, Fiocco M, Mensink G, van Merkesteyn JP. Success and survival of autotransplanted premolars and molars during short-term clinical follow-up. J Clin Periodontol 2016;43:167-172.

3. Yoshino K, Kariya N, Namura D, Noji I, Mitsuhashi K, et al. Influence of age on tooth autotransplantation with complete root formation. $J$ Oral Rehabil 2013;40:112-118.
4. Bae JH, Choi YH, Cho BH, Kim YK, Kim SG. Autotransplantation of teeth with complete root formation: a case series. J Endod 2010;36:14221426.

5. Nagori SA, Bhutia O, Roychoudhury A, Pandey RM. Immediate autotransplantation of third molars: and experience of 57 cases. Oral Surg Oral Med Oral Pathol Oral Radiol 2014;118:400-407. 